\title{
A New Ferric Ion-Selective Fluorescent Chemosensor with a Wide Dynamic Range
}

\author{
Tielong Gao, Kyung Mi Lee, Jiyoung Heo, ${ }^{*}$ and Sung Ik Yang* \\ Department of Applied Chemistry, Kyung Hee University, Yongin 449-701, Korea \\ *E-mail: jiyoung.heo@gmail.com (J.H.), siyang@khu.ac.kr (S.I.Y.) \\ Received March 19, 2010, Accepted May 29, 2010
}

Key Words: Fluorescent chemosensor, Rhodamine, Ferric ion

Iron is an essential metal that plays an important role in numerous biological processes as an element of various metalloproteins. ${ }^{1}$ Many enzymatic reactions involving iron are related to redox reaction in numerous cellular functions such as the oxygen transport of hemoglobin and the electron transfer of heme-containing cytochromes. ${ }^{1}$ The excess of iron, however, can induce various disorders including some neurodegenerative diseases such as Alzheimer's and Parkinson's disease which are related to the iron accumulation in brain. ${ }^{2}$ Hence, the tracking of ferric ion is crucial for studying the physiological and pathological roles of ferric ions in living systems. The development of monitoring methods of iron ions is also required on the environmental aspects since the corrosive water supply pipes could contain higher level of iron ions. The United States Environmental Protection Agency (U.S. EPA) sets the secondary contaminant level for iron as 300 ppb in drinking water. ${ }^{3}$ Although the iron is not health threatening at this level, it can cause undesirable tastes or color and is also one of typical metals responsible for corrosion. Therefore, development of efficient iron sensors with a wider detection range might be highly desirable for real time environmental monitoring.

Design of fluorescent chemosensors for selective detection of target metal ions is an area of intense research activity, since they can provide the potential for real time monitoring with superb sensitivity, selectivity, and high spatial resolution. Since iron ion is paramagnetic, many iron sensors gave rise to the fluorescence-quenching process, ${ }^{4,5}$ although the measurement of fluorescence enhancement upon binding with an analyte is more efficient.

The Rhodamine-based fluorescent chemosensors for detecting metal ions have attracted increasing interests since the binding of specific metal ions leads to non-fluorescent spirolactam opening bringing on color appearance and increase in fluorescence intensity. ${ }^{6}$ Several Rhodamine-based sensors selective to $\mathrm{Fe}^{3+}$ have been reported..$^{7-9}$ As an effort for the design of highly selective and sensitive fluorescent sensor for $\mathrm{Fe}^{3+}$, we present herein the synthesis and characterization of a new Rhod- amine-based fluoroionophore $\mathbf{R h} \mathbf{3}$, which displays high selectivity for $\mathrm{Fe}^{3+}$ and exhibits the wide detection range in concentration.

Rh3 was prepared according to Scheme 1. Compound $\mathbf{1}$ was synthesized using modified method of Shiraishi et al. ${ }^{10}$ Briefly, Rhodamine B hydrochloride salt was refluxed in $\mathrm{POCl}_{3}$ for $12 \mathrm{~h}$ and concentrated under vacuum. After dissolving in dried $\mathrm{CH}_{3} \mathrm{CN}$, 2-bromoethylamine hydrobromide and triethylamine was added to the solution, and stirred for $20 \mathrm{~h}$ at room temperature. The mixture was concentrated by evaporation, and extracted with ethyl acetate $(20 \mathrm{~mL} \times 3)$, dried over $\mathrm{MgSO}_{4}$, filtered and the solvent was removed. The residue was purified throught the chromatography on silica gel to give compound $\mathbf{1}$. Compound $1(0.55 \mathrm{~g})$ and sodium O-ethyl carbonodithioate $(0.28 \mathrm{~g})$ were stirred in dry dichloromethane overnight at room temperature. The resultant solution was extracted with chloroform, washed with water, dried over $\mathrm{MgSO}_{4}$ and then purified by column chromatography (silica gel, hexane/ethyl acetate $=5 / 1, \mathrm{v} / \mathrm{v})$ to give $\mathbf{R h} \mathbf{3}(0.55 \mathrm{~g})$ as light yellow solid with $77 \%$ yield. ${ }^{11}$

Figure 1 shows the absorption and fluorescence spectra of Rh3 $(10 \mu \mathrm{M})$ in the absence and the presence of various metal ions $\mathrm{Ag}^{+}, \mathrm{Cd}^{2+}, \mathrm{Cu}^{2+}, \mathrm{Fe}^{3+}, \mathrm{Hg}^{2+}, \mathrm{Mn}^{2+}, \mathrm{Na}^{+}, \mathrm{Ni}^{2+}, \mathrm{Pb}^{2+}$, and $\mathrm{Zn}^{2+}$ ) in ethanol. Neither significant absorption in $450 \sim 650 \mathrm{~nm}$ nor fluorescence emission in $535 \sim 650 \mathrm{~nm}\left(\lambda_{\mathrm{ex}}=525 \mathrm{~nm}\right)$ was observed for the free $\mathbf{R h} \mathbf{3}$, indicating that it exists as a nonfluorescent spirocyclic form in ethanol. Addition of all tested metal ions does not lead to the significant absorption except for $\mathrm{Fe}^{3+}$. Upon addition of $\mathrm{Fe}^{3+}$ (10 equiv), Rh3 displays strong fluorescence emission at $577 \mathrm{~nm}$ (205-fold). Rh3 also exhibits the negligibly small fluorescence response to $\mathrm{Pb}^{2+}$ (13-fold). These spectroscopic data imply high selectivity of $\mathbf{R h} \mathbf{3}$ to $\mathrm{Fe}^{3+}$.

The fluorescence response of $\mathbf{R h} \mathbf{3}$ to $\mathrm{Fe}^{3+}$ ion is further explored by varying the concentration of $\mathrm{Fe}^{3+}$ ion from 0 to 160 equiv (Fig. 2). The fluorescence intensity is increased by 20 -fold at 1.0 equiv and close to saturation at 160 equiv (800fold). This wide range of concentration response suggests the

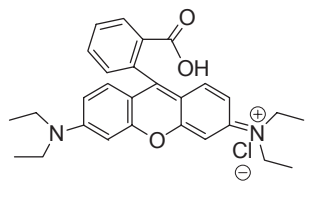

$\underset{\text { 2. } \mathrm{Br} \sim \mathrm{PHCl}_{3}, \text { reflux, } 12 \mathrm{~h}}{\longrightarrow}$

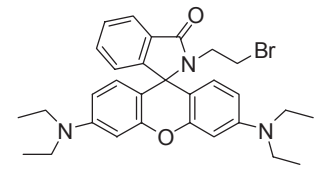

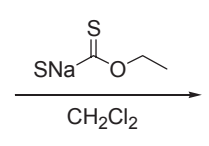

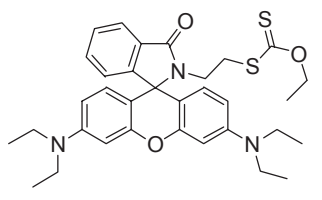

Rh3 $77 \%$

Scheme 1. Synthesis of Rhodamine-based chemosensor $\mathbf{R h} \mathbf{3}$ 

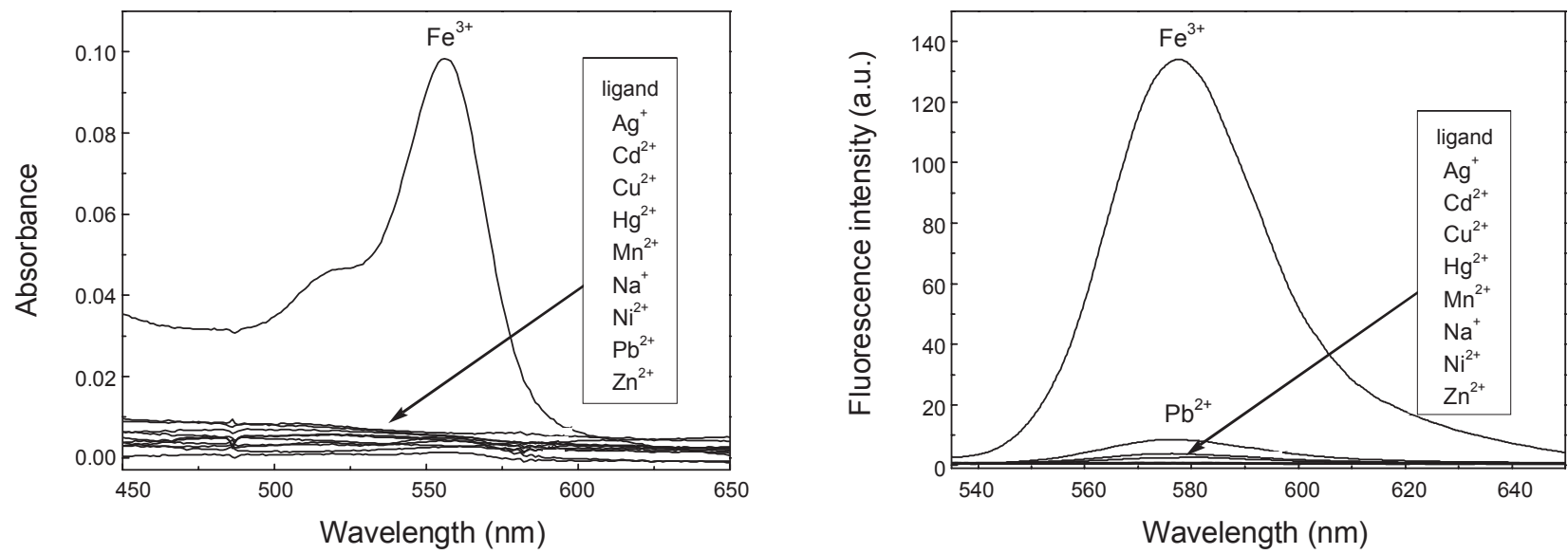

Figure 1. The absorption (left) and fluorescence (right) spectra $\left(\lambda_{\mathrm{ex}}=525 \mathrm{~nm}\right)$ of $\mathbf{R h} \mathbf{3}$ sensor $(10 \mu \mathrm{M})$ in the presence of different metal ions (10 equiv) in ethanol.

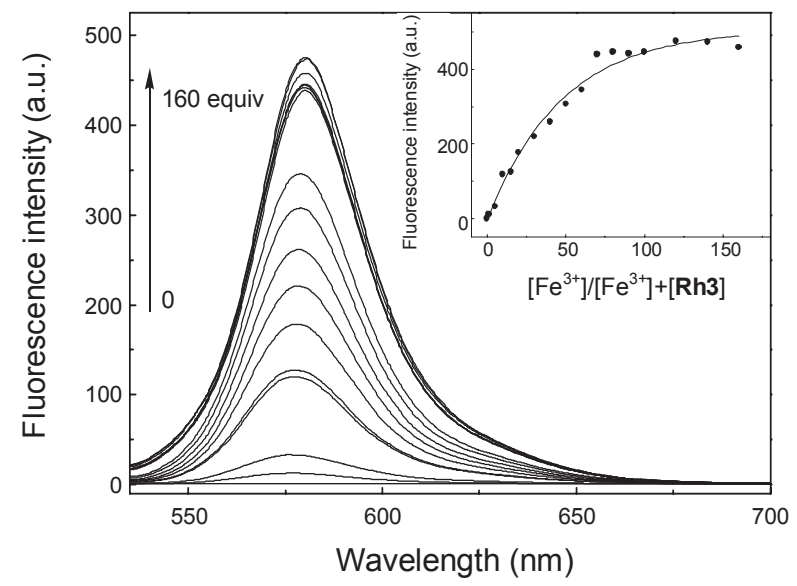

Figure 2. Fluorescence emission spectra of $\mathbf{R h} 3(10 \mu \mathrm{M})$ in the presence of different concentration of $\mathrm{Fe}^{3+}$ in ethanol $(0-160$ equiv). Inset: Plot of fluorescence intensity at $580 \mathrm{~nm}$ as a function of $\left[\mathrm{Fe}^{3+}\right] /$ $\left(\left[\mathrm{Fe}^{3+}\right]+[\mathbf{R h} 3]\right)$.

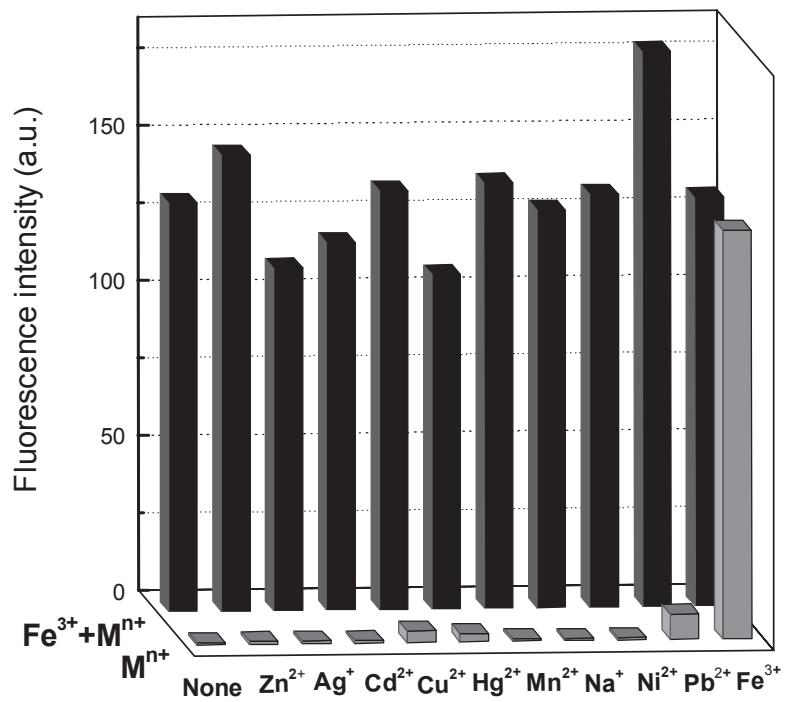

Figure 3. Fluorescence response of $\mathbf{R h} \mathbf{3}$ upon addition of $\mathrm{Fe}^{3+}$ in ethanol under the presence of background metal ions (in the same equivalence of $\mathrm{Fe}^{3+}$ ). Excitation and emission wavelengths are 525 $\mathrm{nm}$ and $580 \mathrm{~nm}$, respectively. use of $\mathbf{R h} \mathbf{3}$ as a sensor with a wide dynamic range. Some fluorescent sensors for a metal ion covering broad range of concentration were previously reported by other groups. Komatus et al. developed a series of fluorescein derivatives, each of which displays a different saturation limit, altogether encompassing a wide detection range on the concentration of a metal ion. ${ }^{12}$ The valuable merit of $\mathbf{R h} \mathbf{3}$ is that with a sensor of a typical concentration, we can detect a $\mathrm{Fe}^{3+}$ ion in $100 \mathrm{ppb}$ to 100 ppm since $\mathbf{R h} \mathbf{3}$ shows high saturation limit and also reasonable sensitivity.

Figure 3 shows the interference of fluorescence response by other metal ions for binding of $\mathrm{Fe}^{3+}$ to $\mathbf{R h} \mathbf{3}$. The background metal ion in the same equivalence to $\mathrm{Fe}^{3+}$ (10 equiv) is added to the $\mathbf{R h} \mathbf{3}$ solution $(10 \mu \mathrm{M})$ along with $\mathrm{Fe}^{3+}$ ion and the fluorescence response is compared to that in the absence of $\mathrm{Fe}^{3+}$. As expected, $\mathrm{Pb}^{2+}$ leads to the slight increase of fluorescence intensity, compared to the $\mathrm{Fe}^{3+}$-alone case since it is under the condition below the saturation limit of a sensor. Any significant interference is not observed for all tested background metal ions, showing high selectivity for $\mathrm{Fe}^{3+}$.

The binding of $\mathrm{Fe}^{3+}$ is expected to be reversible, which was confirmed by the titration with EDTA. The fluorescence of $\mathbf{R h} \mathbf{3}$ $(10 \mu \mathrm{M})$ complex with 10 equiv of $\mathrm{Fe}^{3+}$ disappears in $\sim 5 \mathrm{~min}$ after treatment of excess EDTA.

Based on the above experimental observations, we propose the geometry of $\mathbf{R h} \mathbf{3}$ and $\mathrm{Fe}^{3+}$ complex. Considering the turning-on of absorption and fluorescence in the presence of $\mathrm{Fe}^{3+}$, the spirocyclic ring possibly opens upon binding of the metal to $\mathbf{R h} \mathbf{3}$ as shown in Figure 4. In addition to the carbonyl oxygen, the oxygen and sulfur atoms in the attached unit could make more coordinations with $\mathrm{Fe}^{3+}$. By maximizing the coordination

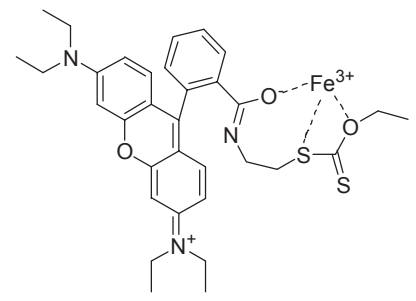

Figure 4. Suggested geometry of $\mathbf{R h} \mathbf{3}$ and $\mathrm{Fe}^{3+}$. 
from $\mathbf{R h} \mathbf{3}$, the 3-coordination geometry is suggested. The remaining coordination sites could be occupied by the solvent oxygen atoms or counter-anions of metal salts.

In conclusion, we have synthesized and characterized a new Rhodamine-based chemosensor $\mathbf{R h} \mathbf{3}$, which is of high sensitivity and of great selectivity toward $\mathrm{Fe}^{3+}$ ion over other metal ions. The Rh3 exhibits the 20 - to 800 -fold increases in fluorescence intensity by the addition of 1 to 160 equiv of $\mathrm{Fe}^{3+}$, presenting the mild fluorescence response. Therefore it could be particularly useful for monitoring $\mathrm{Fe}^{3+}$ ions in broad range of concentrations.

Acknowledgments. This work was supported by a grant from the Kyung Hee University in 2006 (KHU-20060581).

\section{References}

1. Messerschmidt, A.; Huber, R.; Wieghardt, K.; Poulos, T. Handbook of Metalloproteins; Wiley: 2001.

2. Que, E. L.; Domaille, D. W.; Chang, C. J. Chem. Rev. 2008, 108, 1517 .
3. Secondary Drinking Water Regulations: Guidance for Nuisance Chemicals. http://www.epa.gov/ogwdw000/consumer/2ndstandards.html (accessed March 15, 2010).

4. Ma, Y. M.; Luo, W.; Quinn, P. J.; Liu, Z. D.; Hider, R. C. J. Med. Chem. 2004, 47, 6349.

5. Weizman, H.; Ardon, O.; Mester, B.; Libman, J.; Dwir, O.; Hadar, Y.; Chen, Y.; Shanzer, A. J. Am. Chem. Soc. 1996, 118, 12368.

6. Kim, H. N.; Lee, M. H.; Kim, H. J.; Kim, J. S.; Yoon, J. Chem. Soc. Rev. 2008, 37, 1465

7. Xiang, Y.; Tong, A. J. Org. Lett. 2006, 8, 1549.

8. Bae, S.; Tae, J. Tetrahedron Lett. 2007, 48, 5389.

9. Gao, T.; Lee, K. M.; Yang, S. I. Toxicol. Environ. Health Sci. 2010, 2,73 .

10. Shiraishi, Y.; Sumiya, S.; Kohno, Y.; Hirai, T. J. Org. Chem. 2008, 73, 8571 .

11. ${ }^{1} \mathrm{H}-\mathrm{NMR}\left(\mathrm{CDCl}_{3}, 300 \mathrm{MHz}\right), \delta 1.17\left(\mathrm{t}, 12 \mathrm{H}, \mathrm{NCH}_{2} \mathrm{CH}_{3}, J=7.0\right.$ $\mathrm{Hz}), 1.34(\mathrm{t}, 3 \mathrm{H}), 2.86(\mathrm{t}, 2 \mathrm{H}), 3.33(\mathrm{t}, 8 \mathrm{H}), 3.43(\mathrm{~m}, 2 \mathrm{H}), 4.54(\mathrm{q}$, $2 \mathrm{H}), 6.24(\mathrm{dd}, 2 \mathrm{H}$, Xanthene- $\mathrm{H}, J 1=9.0 \mathrm{~Hz}, J 2=2.4 \mathrm{~Hz}), 6.38$ $6.46(\mathrm{~m}, 4 \mathrm{H}), 7.107(\mathrm{~m}, 1 \mathrm{H}), 7.30(\mathrm{~m}, 1 \mathrm{H}), 7.44(\mathrm{~m}, 2 \mathrm{H}), 7.90$ $(\mathrm{m}, 1 \mathrm{H}) .{ }^{13} \mathrm{C}-\mathrm{NMR}\left(\mathrm{CDCl}_{3}\right) \delta 213.4,168.1,153.8,153.2,148.8$, $132.5,130.7,128.8,128.0,123.8,122.8,108.1,105.2,97.7,69.7$, 64.8, 44.3, 39.0, 33.0, 13.7, 12.6. HRMS $\left(\mathrm{EI}^{+}\right) \mathrm{m} / \mathrm{z}$ calcd for $\mathrm{C}_{33} \mathrm{H}_{39} \mathrm{~N}_{3} \mathrm{O}_{3} \mathrm{~S}_{2}:$ 589.2433, Found: 589.2417.

12. Komatsu, K.; Kikuchi, K.; Kojima, H.; Urano, Y.; Nagano, T. J. Am. Chem. Soc. 2005, 127, 10197.s 